\title{
Ultraviolet Absorption Coefficients of Melanosomes Containing Eumelanin As Related to the Relative Content of DHI and DHICA
}

\author{
Dana N. Peles, ${ }^{\dagger}$ Erica Lin, ${ }^{\dagger}$ Kazumasa Wakamatsu, ${ }^{\ddagger}$ Shosuke Ito, ${ }^{\dagger}$ and John D. Simon ${ }^{*}{ }^{\dagger}$ \\ ${ }^{\dagger}$ Department of Chemistry, Duke University, Durham, North Carolina 27708, and ${ }^{\ddagger}$ Department of Chemistry, \\ Fujita Health University School of Health Sciences, Toyoake Aichi 470-1192, Japan
}

\begin{abstract}
Central to understanding the photochemical function(s) of melanosomes is the determination of their absorption properties and an understanding of how the absorption varies with the molecular composition of melanin. Herein, the absorption coefficients for melanosomes containing predominantly eumelanin, a polymeric pigment derived from the molecular precursors 5,6-dihydroxyindole (DHI) and 5,6-dihydroxyindole-2-carboxylic acid(DHICA), are reported for $\lambda=244 \mathrm{~nm}$. The absorption coefficient varies with the DHICA/DHI ratio, determined from chemical degradation analyses. With increasing DHICA content, the absorption coefficient of the melanosome increases. This observation is consistent with reported extinction coefficients, which reveal that at $244 \mathrm{~nm}$, the extinction coefficient of DHICA is a factor of $\sim 2.1$ greater than that of DHI. The melanosome absorption coefficients are compared to predicted values based on a linear combination of the absorption of the constituent monomers.
\end{abstract}

SECTION Biophysical Chemistry

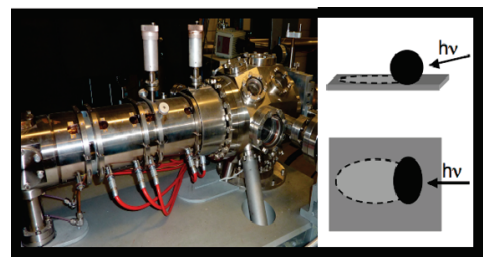

$\mathrm{E}$ umelanin is a commonly occurring pigment derived from the molecular precursors 5,6-dihydroxyindole (DHI) and 5,6-dihydroxyindole-2-carboxylic acid (DHICA). ${ }^{1-4}$ Even though the molecular structure of eumelanin remains elusive, exquisite analytical techniques have been developed that enable determination of the relative contributions of these two precursors to the overall pigment. ${ }^{2,5}$ Studies of eumelanins from different tissues exhibit a wide range of DHICA/DHI ratios. ${ }^{5-8}$ However, how such changes in the molecular composition affect the eumelanic UV absorption coefficient has not been examined for natural systems. An understanding of these effects is critical as photoprotection is often invoked as one of the major biological functions of eumelanin. ${ }^{9,10}$

Approaches to date for determining the optical properties of intact melanosomes, the natural organelles containing eumelanin, involve indirect measurements and require assumptions about the thermodynamic properties of the intact organelle. ${ }^{11-16}$ For example, Jacques et al. ${ }^{15}$ utilized pulsed laser disruption to calculate internal absorption coefficients of several types of melanosomes. Using a pulsed laser to heat melanosomes and analyzing the measured heat with assumptions about melanosomal density and specific heat, they determined the wavelength-dependent melanosome absorption coefficient to be $\varepsilon c(\lambda)=6.49 \times 10^{12}(\lambda)^{-3.58} \mathrm{~cm}^{-1}$. The lack of knowledge about the molecular structure of melanin precludes determination of $c$; therefore, only the product $\varepsilon c$ can be quantified currently. Thus, the melanosome absorption coefficient is reported as the product $\varepsilon c$, which has units of inverse length.
Photoemission electron microscopy (PEEM) can be used to directly quantify the absorption coefficient of single, intact melansomes. ${ }^{17,18}$ Herein, we apply this method to measure the absorption cross sections of natural melanosomes comprised predominantly of eumelanin but of varying DHICA/DHI ratios. ${ }^{7}$ This enables a quantitative examination of the relationship between the absorption coefficients of the intact natural melanosomes and their DHICA/DHI content.

Figure 1 shows representative PEEM images. At the excitation wavelength, both the substrate and the melanosomes photoemit. The melanosomes are brighter, however, because they have a greater photoionization cross section than the titanium substrate. The shape and dimensions of the observed melanosomes are consistent with previously reported scanning electron microscopy analyses. ${ }^{7}$ The substrate reveals shadows on the far side of the melanosomes along the direction of incident light (Figure $1 \mathrm{a}-\mathrm{f}$ ). The shadows reflect $\mathrm{a}$ decreased photoionization intensity of the substrate as a result of the absorption of the incident light by the melanosomes.

An intensity contour plot of the shadow generated by the absorption of $\lambda=244 \mathrm{~nm}$ light by a bovine retinal pigment epithelial melanosome is shown in Figure 2. It is clearly observed that the shadow intensity of the image varies along the direction of the incident light and perpendicular to the

Received Date: May 27, 2010

Accepted Date: July 13, 2010

Published on Web Date: July 19, 2010 

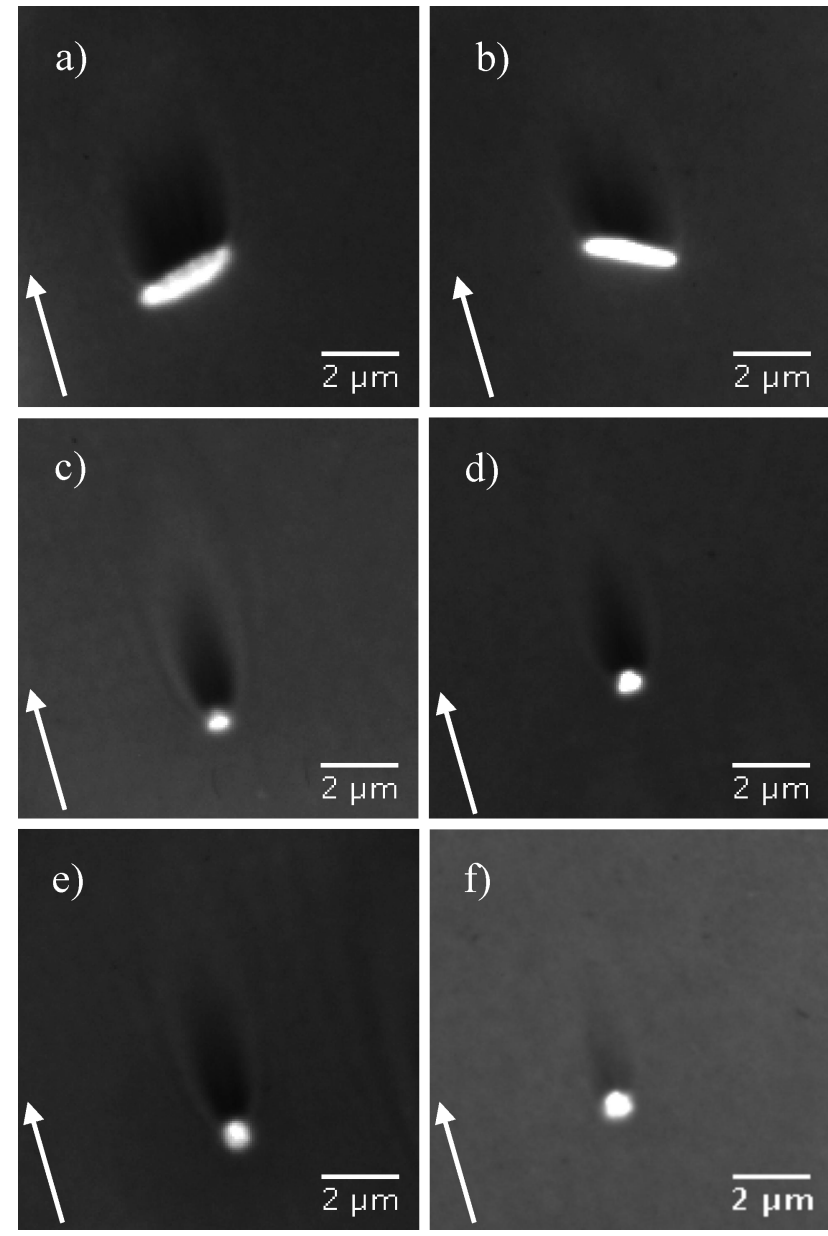

Figure 1. PEEM images of (a) an adult RPE melanosome, (b) a newborn RPE melanosome, (c) adult choroid melanosomes, (d) newborn choroid melanosomes, (e) adult iris melanosomes, and (f) black hair melanosomes. The images clearly reveal the shadows generated as a result of absorption of the incident light. The shadows are oriented in the direction of incident light (indicated by the white arrow).

incident light. This spatial dependence is a result of the different path lengths that light travels through the melanosome. The axial symmetry of the ellipsoidal structure of the melanosomes is reflected in the contour plot of the shadow.

The absorption coefficients, $\varepsilon c$, for single eumelanic melanosomes are calculated as described in the Experimental Methods section; the results are presented in Table 1. The measured $\varepsilon c$ values are consistent with the absorption properties reported for cutaneous melanosomes but nearly an order of magnitude smaller than the values estimated by Jacques et al. ${ }^{15,16}$ The work by Jacques et al. assumes values for the density and specific heat of the melanosome and then uses these values to determine absorption properties through laser-induced heating. These properties have yet to be quantified, and therefore, their data cannot be re-evaluated in terms of independently determined thermodynamic parameters. The work presented herein, however, directly quantifies absorption properties from intact melanosomes independent of such assumptions.

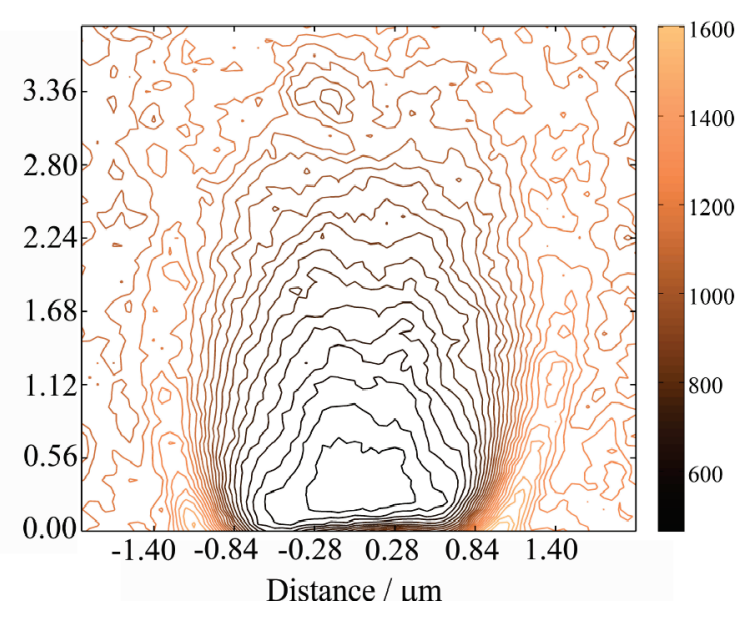

Figure 2. Intensity contour plot of the shadow resulting from a newborn bovine RPE melanosome (not pictured).

Table 1 also includes the DHICA/DHI ratios for the different samples studied as determined through chemical degradation analyses. ${ }^{5-7}$ The decrease in the DHICA/DHI ratio with age is statistically significant and has been discussed in detail in a previous publication. ${ }^{7}$ As the DHICA content increases, the $\varepsilon c$ of the melanosome increases. It is interesting to compare this observation to the UV absorption spectra and extinction coefficients for the DHI and DHICA monomers. ${ }^{19-24}$ The absorption cross sections of DHI and DHICA are somewhat sensitive to $\mathrm{pH}$; an average of the $\varepsilon$ values from the published literature gives 2610 and $5530 \mathrm{M}^{-1} \mathrm{~cm}^{-1}$ (at $\lambda=$ $244 \mathrm{~nm}$ ) for DHI and DHICA, respectively. On the basis of the absorption cross section of the contributing monomers, an increased absorption is expected with increased DHICA/DHI ratios. We note, however, that melanin also contains oxidized indoles, which could affect the absorption coefficient. The percentage of the indoles present in oxidized form(s) has not been established and is likely to be quite small.

If we were to model the absorption of the melanosome as arising from the sum of the absorption of contributing DHI and DHICA monomers, we would write

$$
A=\mathbf{l}(\varepsilon c)_{\text {eumelanin }}=\mathbf{l}\left(\varepsilon_{\mathrm{DHI}}[\mathrm{DHI}]+\varepsilon_{\mathrm{DHICA}}[\mathrm{DHICA}]\right)
$$

In terms of mole fraction, $X$, eq 1 becomes

$$
\varepsilon_{\text {eumelanin }}=\varepsilon_{\mathrm{DHI}} X_{\mathrm{DHI}}+\varepsilon_{\mathrm{DHICA}} X_{\mathrm{DHICA}}
$$

Because the total mole fraction is unity, $1-X_{\mathrm{DHCAI}}=X_{\mathrm{DHI}}$, and eq 1 can be written as

$$
\varepsilon_{\text {eumelanin }}=X_{\mathrm{DHICA}}\left(\varepsilon_{\mathrm{DHICA}}-\varepsilon_{\mathrm{DHI}}\right)+\varepsilon_{\mathrm{DHI}}
$$

Under this assumption, a plot of the melanosome absorption coefficients as a function of the percentage of DHICA content would have limiting values at the end points $X_{\text {DHICA }}=0$ and 1 , given by $\varepsilon_{\mathrm{DHI}}$ and $\varepsilon_{\mathrm{DHICA}}$, respectively. Figure 3 shows a plot of $\varepsilon c$ as a function of the mole fraction of DHICA contained in the melanosomes. As mentioned in the experimental details, the PEEM technique is unable to provide an accurate determination of the size dimensions of each individual melanosome, and therefore, the average size dimensions are used for each melanosome. The standard deviation for the value of the 
Table 1. Summary of the Previously Reported ${ }^{6,7}$ Chemical Degradation Analyses $^{a}$ of Eumelanic Melanosomes with the $\varepsilon c$ Analysis Reported Herein

\begin{tabular}{llcccccc}
\hline melanosomes & age & $\mathrm{PTCA}^{b} \mu \mathrm{g} / \mathrm{mg}$ & $\mathrm{TM} \mathrm{A}_{500} / \mathrm{mg}$ & $\mathrm{PTCA} / \mathrm{TM} \mu \mathrm{g} / \mathrm{A}_{500}$ & DHICA/DHI & $\varepsilon c\left(\mathrm{~cm}^{-1}\right)$ & no. of melanosomes pooled \\
\hline \multirow{2}{*}{$\mathrm{RPE}$} & newborn & 7.7 & 5.91 & 1.30 & $1.30: 1$ & 5270 & 71 \\
& adult & 4.2 & 5.26 & 0.80 & $0.80: 1$ & 3655 & 34 \\
\multirow{2}{*}{ choroid } & newborn & 14.7 & 6.34 & 2.32 & $2.32: 1$ & 5115 & 64 \\
& adult & 5.6 & 4.60 & 1.21 & $1.21: 1$ & 5085 & 58 \\
\multirow{2}{*}{ iris } & adult & 5.2 & 5.19 & 1.00 & $1.00: 1$ & 5175 & 37 \\
black hair & & 1.5 & 5.80 & 0.26 & $0.26: 1$ & 3695 & 50
\end{tabular}

${ }^{a}$ Chemical degradation products are expressed in units of per mg melanosomes. ${ }^{b}$ Determined from oxidation by $\mathrm{KMnO}_{4}$.

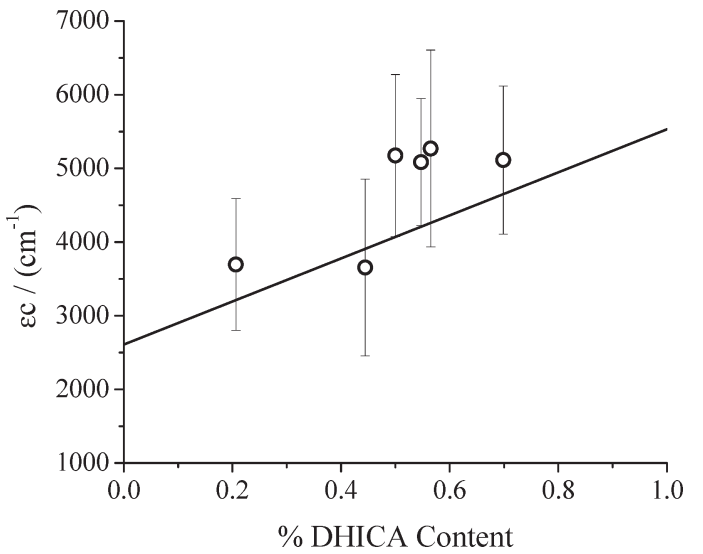

Figure 3. Plot of the observed $\varepsilon c$ versus DHICA content. The line is representative of the predicted absorption as arising from the sum of the absorption of contributing DHI and DHICA monomers.

absorption coefficient is then determined by propagation of the error from the size analyses of the melanosomes. The error in determining the percentage DHICA content is $<7 \%$. The line shown in the plot is drawn between the average $\varepsilon$ values of the two monomers ( 2610 and $5530 \mathrm{M}^{-1} \mathrm{~cm}^{-1}$ ). This line represents the predicted absorption coefficients, assuming that this value is given by the sum of the constituent concentration of contributing DHI and DHICA monomers, eq 1 . The observed $\varepsilon c$ are consistent with the trend predicted by this model. These results provide support that the absorption of natural melanins can be quantified in terms of relative content of the two constituent monomers, weighted by their relative mole fractions.

The structures of natural oligomers formed from DHI and DHICA are not known; even the soluble fraction of melanins has proven difficult to characterize by molecular structure techniques such as mass spectrometry. Recently, papers ${ }^{20,21,25-27}$ have appeared that provide insight into the absorption properties of synthetic oligomers of DHI and DHICA. However, the collective work does not provide insight into how the absorption properties of the oligomer structure (dimer to tetramer) are related to the cross sections of the monomeric building blocks. For examples, in a study of oligomers of DHI, $\mathrm{d}^{\prime}$ Ischia and co-workers ${ }^{20}$ find that for some structures, the absorption coefficient at $\sim 250 \mathrm{~nm}$ roughly scales with the number of monomeric units, whereas for others, the absorption spectrum is insensitive to the number of monomers. A study by Reisz et al. ${ }^{27}$ suggests a $20 \%$ hyperchromism in the
DHICA polymer compared to that in the monomer. Thus, the spectroscopic properties of oligomers produced from these building blocks depend on the chemical linkages between units. The results obtained herein on natural systems suggest that the absorption coefficient scales linearly with the compositional ratio of the two monomeric units, which ultimately may be a reflection of the naturally occurring oligomers that define the structure of eumelanin. The absorption data on intact melanosomes therefore offers new insights into distinguishing possible oligomeric arrangements of DHI and DHICA that could be present in natural systems.

In mammals, the DHICA/DHI ratio can be increased by the action of dopachrome tautomerase (Dct). ${ }^{28}$ Mice with a wildtype Dct produce black hair with a DHICA/DHI ratio of $\sim 3$, while a mutation of the Dct gene leads to production of slaty hair with a DHICA/DHI ratio of $\sim 0.3 .^{29}$ Although how the increased ratio of DHICA in eumelanin is beneficial to animals is not well elaborated, the increased light absorption in the UV region by melanosomes with high DHICA/DHI ratios should contribute to absorption of harmful UV light. It should also be pointed out that synthetic DHICA-melanin exhibits a potent hydroxyl radical scavenging effect as compared with DHImelanin. 30

The shapes of the absorption spectra of DHI and DHICA are similar in the region from $220-270 \mathrm{~nm}$, although DHICA has a 3-4-fold greater cross- section. The spectra of the two monomers differ significantly in shape in the UV-A, the absorption maxima (extinction coefficient) being $296 \mathrm{~nm}$ $\left(3310 \mathrm{M}^{-1} \mathrm{~cm}^{-1}\right)$ and $2320 \mathrm{~nm}\left(\sim 14000-16000 \mathrm{M}^{-1} \mathrm{~cm}^{-1}\right)$, respectively. $^{21,22}$ This means that the region from 300 to 400 $\mathrm{nm}$ would be an excellent range to examine whether $\varepsilon c$ for the melanosome maintains an additive relationship with the constituent monomers across the absorption spectrum. Reported spectra on small synthetic oligomers reveal that this lower-energy region is more sensitive to the oligomer structure than the region around $\sim 250 \mathrm{~nm}$, and therefore, it is important to determine the full absorption spectrum of the natural melanosome. Work to extend the PEEM approach to accomplish this goal is in progress and will be reported at a later date.

\section{EXPERIMENTAL METHODS}

Sample Isolation and Preparation. Details of the isolation of the studied melanosomes from bovine RPE cells, bovine iris and choroid tissues, and black human hair were previously 
published along with their chemical analyses. ${ }^{6,7}$ The chemical analyses enabled the determination of the relative DHI and DHICA content. Additionally, the melanosome preparation for PEEM studies has been previously published. ${ }^{18}$

Photoemission Electron Microscopy. Details of the Elmitec ultrahigh vacuum PEEM used in this study have been previously described in detail. ${ }^{31}$ Laser excitation of the sample at $\lambda=244 \mathrm{~nm}(5.07 \mathrm{eV})$ was provided by an intracavity doubled $\mathrm{Ar}^{+}$ion laser. A zero-order half-wave plate was used to orient the polarization of the light so that it was parallel to the plane of incidence.

Determination of the Absorption Coefficient from the PEEM Image. We recently described the details of calculating the absorption coefficient, $\varepsilon c$, from the PEEM image. ${ }^{18}$ Starting with the Beer-Lambert law

$$
\varepsilon c=A l^{-1}=l^{-1} \log \left(I_{0} / I\right)
$$

the calculation of $\varepsilon c$ requires knowledge of the path length, $l$, and the incident and transmitted intensities of light, $I_{0}$ and $I$, respectively. $I$ and $I_{0}$ are obtained from the PEEM image; an average of the photoemission current of the substrate gives $I_{0}$, and an average of the photoemission current of the shadow region adjacent to the melanosomes gives $I$. As previously reported, ${ }^{18}$ the portion of the shadow that is void of significant scattering contribution is identified as the first minimum of the diffraction pattern produced on the substrate. This region is identified in the images studied herein, and an average value of the transmitted light intensity is subsequently obtained after averaging the intensities of the included pixels.

The spatial dependence of the photoemission current of the shadow maps directly onto the different path lengths through the melanosome, and therefore, the numerical results obtained depend on the determination of the path lengths corresponding to the image. We demonstrated ${ }^{18}$ that the average path length, $\overline{\mathrm{s}}$, through half of the ellipsoidal melanosome is given by

$\overline{\mathrm{S}}=\frac{\int_{0}^{c} \int_{0}^{\left[a^{2}-\left(a^{2} z^{2}\right) / c^{2}\right]^{1 / 2}} 2\left\{b^{2}-\left[\left(b^{2} x^{2}\right) / a^{2}\right]-\left[\left(b^{2} z^{2}\right) / c^{2}\right]\right\}^{1 / 2} \mathrm{~d} x \mathrm{~d} z}{\int_{0}^{c} \int_{0}^{\left[a^{2}-\left(a^{2} z^{2}\right) / c^{2}\right]^{1 / 2}} \mathrm{~d} x \mathrm{~d} z}$

where $a$ and $b$ are the equatorial radii along the $x$ - and $y$-axes, respectively, and $c$ is the polar radius along the $z$-axis. We note, however, that portions of the shadow map onto path lengths that extend beyond half of the volume of the melanosome, the extent to which is determined by the geometry of the experiment. The polar distance, $\chi$, that extends below the center of the melanosome along the $z$-axis, normal to the substrate, can be obtained directly from the PEEM image. Equation 5 then gives the averaged experimental path length when using the integration bounds $z \in[\chi, c]$ and $x \in\left[-\left[a^{2}-\left(a^{2} z^{2}\right) / c^{2}\right]^{1 / 2}\right.$, $\left.\left[a^{2}-\left(a^{2} z^{2}\right) / c^{2}\right]^{1 / 2}\right]$.

The above analysis is accurate for ellipsoids whose long axis is oriented perpendicular to the direction of incident light. However, in the PEEM experiments, melanosomes are oriented at all angles with respect to the direction of incident light. In the case of many of the eumelanosomes studied herein, the aspect ratio is such that the orientation of the melanosomes can be determined from the PEEM image. In these instances, it is necessary to take into account the angle between the incident light and the long axis of the melanosome. The modeled melanosome, originally oriented perpendicular to the incident radiation, is rotated in a counterclockwise rotation around the $z$-axis by $\theta$ radians through variable substitution

$$
\begin{aligned}
& x=X \cos (\theta)+Y \sin (\theta) \quad \text { and } \\
& y=Y \cos (\theta)-X \sin (\theta)
\end{aligned}
$$

The path lengths through the rotated melanosome can then be described by

$$
\begin{gathered}
s(X, Z, \theta) \\
=2\left\{\left[a ^ { 2 } b ^ { 2 } \left[-c^{2} X^{2} \cos (\theta)^{4}+\cos (\theta)^{2}\left[a^{2}\left(c^{2}-z^{2}\right)\right.\right.\right.\right. \\
\left.-2 c^{2} X^{2} \sin (\theta)^{2}\right]-\sin (\theta)^{2}\left[b^{2}\left(-c^{2}+z^{2}\right)\right. \\
\left.\left.\left.\left.+c^{2} X^{2} \sin (\theta)^{2}\right]\right]\right] /\left[c^{2}\left[a^{2} \cos (\theta)^{2}+b^{2} \sin (\theta)^{2}\right]^{2}\right]\right\}^{1 / 2}
\end{gathered}
$$

Note that in this orientation, the major radius no longer lies along the $x$-axis, and as a consequence, the upper limit of the integration bounds for the $X$ variable becomes $\left\{\left[\left(c^{2}-z^{2}\right)\left[a^{2}\right.\right.\right.$ $\left.\left.\left.\cos (\theta)^{2}+b^{2} \sin (\theta)^{2}\right]\right] / c^{2}\right\}^{1 / 2} /\left\{[\cos (2 \theta)]^{2}\right\}^{1 / 2}$. Thus, the average path length for half of the volume of a rotated ellipsoidal melanosome is

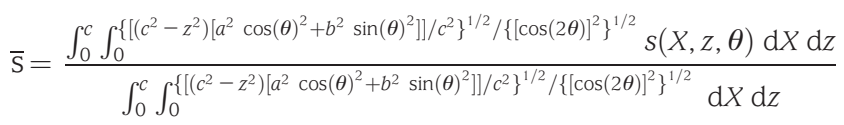

Once again, the polar distance extending below the center of the melanosome for regions that contribute to the shadow can be obtained directly from the PEEM image, and then, the limits of the integrals in eq 8 can be adjusted accordingly.

\section{AUTHOR INFORMATION}

\section{Corresponding Author:}

*To whom correspondence should be addressed. Email: john.simon@ duke.edu.

ACKNOWLEDGMENT This research was supported by Duke University. We thank Dr. Lian Hong for the sample isolation and size analysis data and Professor Robert J. Nemanich for use of the photoemission electron microscope and the UV argon ion laser. We also thank Dr. Robert Guenther for his many discussions and insight into the diffraction of tilted elliptical apertures.

\section{REFERENCES}

(1) Ito, S.; Wakamatsu, K. Chemistry of Mixed Melanogenesis Pivotal Roles of Dopaquinone. Photochem. Photobiol. 2008, 84, 582-592.

(2) Wakamatsu, K.; Ito, S. Advanced Chemical Methods in Melanin Determination. Pigment Cell Res. 2002, 15, 1740-1183.

(3) Ito, S. Reexamination of the Structure of Eumelanin. Biochim. Biophys. Acta 1986, 883, 155-161.

(4) Tsukamoto, K.; Palumbo, A.; D'Ischia, M.; Hearing, V. J.; Prota, G. 5,6-Dihydroxyindole-2-Carboxylic Acid Is 
Incorporated in Mammalian Melanin. Biochem. J. 1992, 286, 491-495.

(5) Ozeki, H.; Wakamatsu, K.; Ito, S.; Ishiguro, I. Chemical Characterization of Eumelanins with Special Emphasis on 5,6-Dihydroxyindole-2-carboxylic Acid Content and Molecular Size. Anal. Biochem. 1997, 248, 149-157.

(6) Liu, Y.; Hong, L.; Wakamatsu, K.; Ito, S.; Adhyaru, B.; Cheng, C.-Y.; Bowers, C. R.; Simon, J. D. Comparison of Structural and Chemical Properties of Black and Red Human Hair Melanosomes. Photochem. Photobiol. 2005, 81, 135-144.

(7) Liu, Y.; Hong, L.; Wakamatsu, K.; Ito, S.; Adhyaru, B. B.; Cheng, C.-Y.; Bowers, C. R.; Simon, J. D. Comparisons of the Structural and Chemical Properties of Melanosomes Isolated from Retinal Pigment Epithelium, Iris and Choroid of Newborn and Mature Bovine Eyes. Photochem. Photobiol. 2005, 81, 510-516.

(8) Liu, Y.; Hong, L.; Kempf, V. R.; Wakamatsu, K.; Ito, S.; Simon, J. D. Ion-Exchange and Adsorption of Fe(III) by Sepia Melanin. Pigment Cell Res. 2004, 17, 262-269

(9) Sarna, T. Properties and Function of the Ocular Melanin - A Photobiophysical View. J. Photochem. Photobiol., B 1992, 12, 215-258.

(10) Sarna, T.; Swartz, H. A. The Physical Properties of Melanins. In The Pigmentary System: Physiology and Pathophysiology; Nordlund, J. J., Boissy, R. E., Hearing, V. J., King, R. A., Oetting, W. S., Ortonne, J.-P., Eds.; Blackwell Publishing: Oxford, U.K., 2006; pp 311-341.

(11) Sardar, D. K.; Mayo, M. L.; Glickman, R. D. Optical Characterization of Melanin. J. Biomed. Opt. 2001, 6, 404-411.

(12) Norvang, L. T.; Fiskerstrand, E. J.; Nelson, J. S.; Berns, M. W.; Svaasand, L. O. Epidermal Melanin Absorption in Human Skin. SPIE Int. Soc. Opt. Eng. 1996, 2624, 143-154.

(13) Neumann, J.; Brinkmann, R. Boiling Nucleation on Melanosomes and Microbeads Transiently Heated by Nanosecond and Microsecond Laser Pulses. J. Biomed. Opt. 2005, 10, 024001

(14) Glickman, R. D.; Jacques, S. L.; Hall, R. T.; Kumar, N. Revisiting the Internal Absorption Coefficient of the Retinal Pigment Epithelium Melanosome. SPIE Int. Soc. Opt. Eng. 2001, 4257, 134-141.

(15) Jacques, S. L.; Glickman, R. D.; Schwartz, J. A. Internal Absorption Coefficient and Threshold for Pulsed Laser Disruption of Melanosomes Isolated from Retinal Pigment Epithelium. SPIE Int. Soc. Opt. Eng. 1996, 2681, 468-477.

(16) Jacques, S. L.; McAuliffe, D. J. The Melanosome: Threshold Temperature for Explosive Vaporization and Internal Absorption Coefficient during Pulsed Laser Irradiation. Photochem. Photobiol. 1991, 53, 769-775.

(17) Peles, D. N.; Simon, J. D. Direct Measurement of the Ultraviolet Absorption Coefficient of Single Retinal Melanosomes. Photochem. Photobiol. 2010, 86, 279-281.

(18) Peles, D. N.; Simon, J. D. The Ultraviolet Absorption Coefficient of Melanosomes Decreases with Increasing Pheomelanin Content. J. Phys. Chem. B 2010, doi: 10.1021/ jp102603b.

(19) Gauden, M.; Pezzella, A.; Panzella, L.; Napolitano, A.; d'Ischia, M.; Sundström, V. Ultrafast Excited State Dynamics of 5,6-Dihydroxyindole, A Key Eumelanin Building Block: Nonradiative Decay Mechanism. J. Phys. Chem. B 2009, 113, 12575-12580.

(20) d'Ischia, M.; Crescenzi, O.; Pezzella, A.; Arzillo, M.; Panzella, L.; Napolitano, A.; Barone, V. Structural Effects on the Electronic Absorption Properties of 5,6-Dihydroxyindole Oligomers: The Potential of an Integrated Experimental and DFT
Approach to Model Eumelanin Optical Properties. Photochem. Photobiol. 2008, 84, 600-607.

(21) Tran, M. L.; Powell, B. J.; Meredith, P. Chemical and Structural Disorder in Eumelanins: A Possible Explanation for Broadband Absorbance. Biophys. J. 2006, 90, 743-752.

(22) Murphy, B. P.; Schultz, T. M. Synthesis and Physical Properties of 5,6-Dihydroxyindole. J. Org. Chem. 1985, 50, 2790-2791.

(23) Ito, S.; Nicol, J. A. C. Isolation of Oligomers of 5,6-Dihydroxyindole-2-carboxylic Acid from the Eye of the Catfish. Biochem. J. 1974, 143, 207-217.

(24) Benigni, J. D.; Minnis, R. L. The Synthesis of 5,6-Dihydroxyindole and Some of Its Derivatives. J. Heterocycl. Chem. 1965, 2, 387-392.

(25) Pezzella, A.; Iadonisi, A.; Valerio, S.; Panzella, L.; Napolitano, A.; Adinolfi, M.; d'Ischia, M. Disentangling Eumelanin "Black Chromophore": Visible Absorption Changes As Signatures of Oxidation State- and Aggregation-Dependent Dynamic Interactions in a Model Water-Soluble 5,6-Dihydroxyindole Polymer. J. Am. Chem. Soc. 2009, 131, 15270-15275.

(26) Pezzella, A.; Panzella, L.; Crescenzi, O.; Napolitano, A.; Suppiah, N.; Edge, R.; Land, E. J.; Barone, V.; D'Ischia, M. Lack of Visible Chromophore Development in the Pulse Radiolysis Oxidation of 5,6-Dihydroxyindole-2-carboxylic Acid Oligomers: DFT Investigation and Implications for Eumelanin Absorption Properties. J. Org. Chem. 2009, 74, 3727-3734

(27) Riesz, J. J.; Gilmore, J. B.; McKenzie, R. H.; Powell, B. J.; Pederson, M. R.; Meredith, P. Transition Dipole Strength of Eumelanin. Phys. Rev. E 2007, 76, 021915.

(28) Kroumpouzos, G.; Urabe, K.; Kobayashi, T.; Sakai, C.; Hearing, V. J. Functional Analysis of the Slaty Gene Product (TRP2) as Dopachrome Tautomerase and the Effect of a Point Mutation on its Catalytic Function. Biochem. Biophys. Res. Commun. 1994, 202, 1060-1068.

(29) Lamoreux, M. L.; Wakamatsu, K.; Ito, S. Interaction of Major Coat Color Gene Functions in Mice as Studied by Chemical Analysis of Eumelanin and Pheomelanin. Pigment Cell Res. 2001, 14, 23-31

(30) Jiang, S.; Liu, X.-M.; Dai, X.; Zhou, Q.; Lei, T.-C.; Beermann, F.; Wakamatsu, K.; Xu, S.-Z. Regulation of DHICA-Mediated Antioxidation by Dopachrome Tautomerase: Implication for Skin Photoprotection Against UVA Radiation. Free Radical Biol. Med. 2010, 48, 1144-1151.

(31) Ade, H.; Yang, W.; English, S. L.; Hartman, J.; Davis, R. F.; Nemanich, R. J.; Litvinenko, V. N.; Pinayev, I. V.; Wu, Y.; Madey, J. M. J. A Free Electron Laser-Photoemission Electron Microscope System (FEL-PEEM). Surf. Rev. Lett. 1998, 5, 1257-1268. 